\title{
Determination of critical plane and assessment of fatigue durability for multiaxial cyclic loading and arbitrary shift of phases
}

\author{
Boris A. Stratula ${ }^{1,3}$, Nikolai G. Burago ${ }^{2}$, Alexander D. Nikitin ${ }^{1,1}$, and Ilia S. Nikitin ${ }^{1}$ \\ ${ }^{1}$ Institute of Computer Aided Design of RAS, Mechanics of RAS, Moscow, Russia \\ ${ }^{2}$ Ishlinsky Institute for Problems in Mechanics of RAS, Moscow, Russia \\ ${ }^{3}$ Moscow Aviation Institute (National Research University), Moscow, Russia
}

\begin{abstract}
An analytical solution for the well-known fatigue criterion proposed by Papadopoulos was obtained. An arbitrary shift of phases was taken into account. Comparison between analytical, numerical solutions with experimental data was made. Computation results based on FEM were compared with assessments by the mentioned above criterion.
\end{abstract}

\section{Introduction}

It is shown by operating experience of various structural elements that natural cyclic regimes and loading conditions can rarely be reproduced in laboratories in simplified fatigue experiments such as tension, bending or torsion. Typically, structural elements are subject to complex (triaxial) stressed states. In this case it is necessary to use multiaxial fatigue criteria. Modern fatigue criteria make it possible to evaluate amount of cycles $\mathrm{N}$ to breakdown either of a specimen or structural element (so called fatigue life). Additionally, an arbitrary shift of phases between cyclic stress components and an orientation of a critical plane are taken into account [1-5]. In this paper a procedure to evaluate an orientation of a critical plane (fig. 1) and hence a fatigue life under multiaxial cyclic loadings with an arbitrary shift of phases is developed. The procedure is based on the well-known criterion [2] and is valid for two classic ranges namely for cases of low- (LCF) and high-cycle fatigue $(\mathrm{HCF})$.

\section{Papadopoulos' fatigue criterion}

Let us consider material particle in a uniform stress state described by a stress tensor $\boldsymbol{\sigma}(t)$ that varies cyclic in time. Let us further choose a frame that is associated with the principal stresses $\sigma_{1}, \sigma_{2}, \sigma_{3}$ and choose a plane with a unit normal $\mathbf{n}$. The multiaxial fatigue criterion is:

$$
\max _{\mathbf{n}} T_{a}+\alpha_{\infty} \sigma_{H, \max }=S_{0}+A N^{\beta}
$$




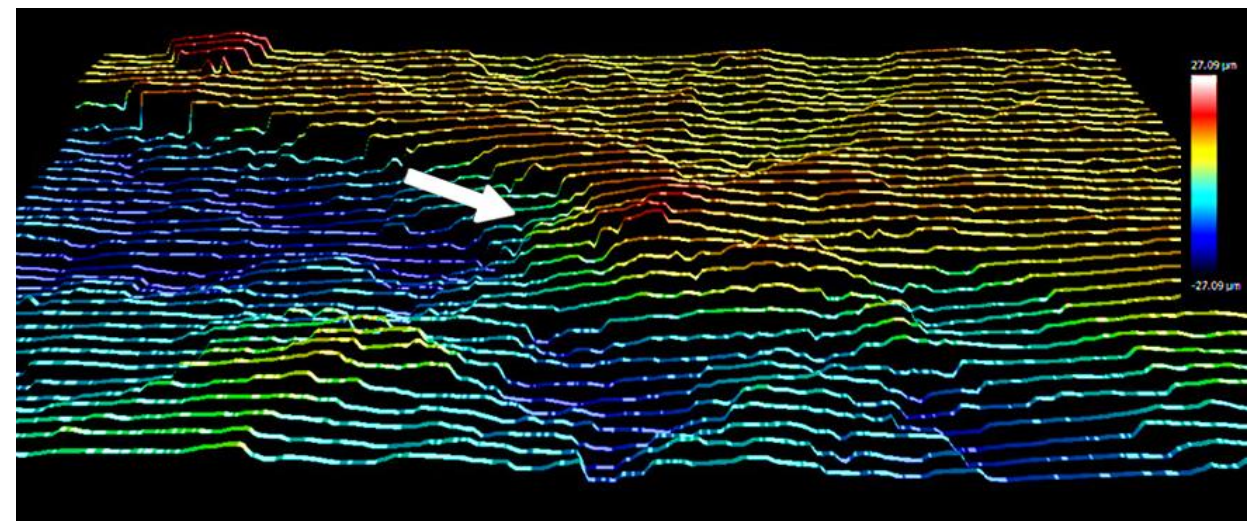

Fig. 1. A critical plane image via CLSM.

Coefficients $S_{0}, A, \alpha_{\infty}$ and $\beta$ are defined by tension-compression fatigue tests with two different asymmetry ratios $R=0$ and $R=-1$ [6], $N$ is the number of cycles to fracture. The quantity $\max T_{a}$ is a maximal value of a shear stress among all existing planes with normals $\mathbf{n}$ that contain the specified material particle in a loading cycle:

$$
\begin{gathered}
T_{a}^{2}(\mathbf{n})=\int_{0}^{2 \pi} \tau_{a}^{2}(\mathbf{n}, \chi) d \chi / \pi \\
\tau_{a}(\mathbf{n}, \chi)=\left[\max _{t \in P} \tau(\mathbf{n}, \chi, t)-\min _{t \in P} \tau(\mathbf{n}, \chi, t)\right]
\end{gathered}
$$

The quantity $\sigma_{H, \max }$ is an equivalent peak hydrostatic stress at the specific place within a loading cycle:

$$
\sigma_{H, \max }=\max _{t \in P} \sigma_{k k}(t) / 3
$$

Shear stress on the plane with normal vector $\mathbf{n}$ is:

$$
\boldsymbol{\tau}=(\boldsymbol{\sigma} \cdot \mathbf{n})-(\mathbf{n} \cdot \boldsymbol{\sigma} \cdot \mathbf{n}) \mathbf{n}
$$

The following equation may be obtained for the value of shear stress spread [7]:

$$
T_{a}^{2}(\mathbf{n})=|\Delta \tau|^{2}=\left(\Delta \sigma_{1}-\Delta \sigma_{2}\right)^{2} n_{1}^{2} n_{2}^{2}+\left(\Delta \sigma_{1}-\Delta \sigma_{3}\right)^{2} n_{1}^{2} n_{3}^{2}+\left(\Delta \sigma_{2}-\Delta \sigma_{3}\right)^{2} n_{2}^{2} n_{3}^{2}
$$

\section{Particular loading cases}

\subsection{Tri-axial tension-compression case}

Let us assume that principal stresses are varied in time by harmonic law with frequency $\omega$ and arbitrary shifts of phase $\varphi_{2}, \varphi_{3}$ :

$$
\sigma_{1}=\sigma_{1 m}+\sigma_{1 a} \cos \omega t
$$




$$
\begin{aligned}
& \sigma_{2}=\sigma_{2 m}+\sigma_{2 a} \cos \left(\omega t+\varphi_{2}\right) \\
& \sigma_{3}=\sigma_{3 m}+\sigma_{3 a} \cos \left(\omega t+\varphi_{3}\right)
\end{aligned}
$$

where $\sigma_{1 m}, \sigma_{2 m}, \sigma_{3 m}$ are mean principal stress components and $\sigma_{1 a}, \sigma_{1 a}, \sigma_{1 a}$ are amplitudes of these stress components. Spreads of the principal stress tensor components within a loading cycle are:

$$
\begin{gathered}
\Delta \sigma_{1}=\sigma_{1 a}\left(\cos \omega t_{1}-\cos \omega t_{2}\right) \\
\Delta \sigma_{2}=\sigma_{2 a}\left(\cos \left(\omega t_{1}+\varphi_{2}\right)-\cos \left(\omega t_{2}+\varphi_{2}\right)\right) \\
\Delta \sigma_{3}=\sigma_{3 a}\left(\cos \left(\omega t_{1}+\varphi_{3}\right)-\cos \left(\omega t_{2}+\varphi_{3}\right)\right)
\end{gathered}
$$

where time instants $t_{1}$ and $t_{2}$ correspond to minimum and maximum values of shear stress at the plane with unit normal vector $\mathbf{n} ; t_{1}, t_{2} \in[0, T], T=2 \pi / \omega$.

The shear stress range can be written as:

$$
\begin{gathered}
\Delta \tau^{2}=4\left[A_{12}^{2} \sin ^{2}\left(\omega \xi-\psi_{12}\right) n_{1}^{2} n_{2}^{2}+A_{13}^{2} \sin ^{2}\left(\omega \xi-\psi_{13}\right) n_{1}^{2} n_{3}^{2}+A_{23}^{2} \sin ^{2}\left(\omega \xi-\psi_{23}\right) n_{2}^{2} n_{3}^{2}\right] \\
\frac{t_{1}+t_{2}}{2}=\xi, A_{12}^{2}=\Delta \sigma_{12}^{2}+\tilde{\sigma}_{2 a}^{2}, A_{13}^{2}=\Delta \sigma_{13}^{2}+\tilde{\sigma}_{3 a}^{2}, A_{23}^{2}=\Delta \sigma_{23}^{2}+\Delta \tilde{\sigma}_{23}^{2}, \\
t_{2}=t_{1}+\frac{\pi}{\omega}=t_{1}+T / 2, \psi_{12}=\operatorname{arctg} \frac{\tilde{\sigma}_{2 a}}{\Delta \sigma_{12}}, \psi_{13}=\operatorname{arctg} \frac{\tilde{\sigma}_{3 a}}{\Delta \sigma_{13}}, \psi_{23}=-\operatorname{arctg} \frac{\Delta \tilde{\sigma}_{23}}{\Delta \sigma_{23}},
\end{gathered}
$$

where:

$$
\begin{gathered}
\Delta \sigma_{12}=\sigma_{1 a}-\sigma_{2 a} \cos \varphi_{2}, \Delta \sigma_{13}=\sigma_{1 a}-\sigma_{3 a} \cos \varphi_{3}, \Delta \sigma_{23}=\sigma_{2 a} \cos \varphi_{2}-\sigma_{3 a} \cos \varphi_{3}, \\
\tilde{\sigma}_{2 a}=\sigma_{2 a} \sin \varphi_{2}, \tilde{\sigma}_{3 a}=\sigma_{3 a} \sin \varphi_{3}, \Delta \tilde{\sigma}_{23}=\sigma_{2 a} \sin \varphi_{2}-\sigma_{3 a} \sin \varphi_{3}, \\
A_{S}=A_{12}^{2} \sin 2 \psi_{12} n_{1}^{2} n_{2}^{2}+A_{13}^{2} \sin 2 \psi_{13} n_{1}^{2} n_{3}^{2}+A_{23}^{2} \sin 2 \psi_{23} n_{2}^{2} n_{3}^{2}, \\
A_{C}=A_{12}^{2} \cos 2 \psi_{12} n_{1}^{2} n_{2}^{2}+A_{13}^{2} \cos 2 \psi_{13} n_{1}^{2} n_{3}^{2}+A_{23}^{2} \cos 2 \psi_{23} n_{2}^{2} n_{3}^{2}, \\
b_{1}=A_{13}^{2} \sin ^{2}\left(\omega \xi_{0}-\psi_{13}\right), b_{2}=A_{23}^{2} \sin ^{2}\left(\omega \xi_{0}-\psi_{23}\right), \\
b_{12}=A_{13}^{2} \sin ^{2}\left(\omega \xi_{0}-\psi_{13}\right)+A_{23}^{2} \sin ^{2}\left(\omega \xi_{0}-\psi_{23}\right)-A_{12}^{2} \sin ^{2}\left(\omega \xi_{0}-\psi_{12}\right)
\end{gathered}
$$

Expressions for the time stamps and frequency are:

$$
\begin{gathered}
t_{1}=\frac{T}{4}\left(\frac{\operatorname{arctg}\left(A_{S} / A_{C}\right)}{\pi}+k-1\right), t_{2}=\frac{T}{4}\left(\frac{\operatorname{arctg}\left(A_{S} / A_{C}\right)}{\pi}+k+1\right) \\
\omega \xi_{0}=\frac{\operatorname{arctg}\left(A_{S} / A_{C}\right)}{2}+\frac{\pi k}{2}, \quad k=1,2,3
\end{gathered}
$$


Corresponding values of the unit normal vector's components $x_{i}=n_{i}^{2}$ at the time instants $t_{1}$ and $t_{2}$ were obtained:

$$
x_{1}=\left(2 b_{1} b_{2}-b_{2} b_{12}\right) /\left(4 b_{1} b_{2}-b_{12}^{2}\right), x_{2}=\left(2 b_{1} b_{2}-b_{1} b_{12}\right) /\left(4 b_{1} b_{2}-b_{12}^{2}\right)
$$

In a similar way a time stamp within the loading cycle that determines the greatest value of $\alpha_{\infty} \sigma_{H, \max }$ was found:

$$
\sigma_{H, \max }=\max _{t \in P} \sigma_{k k}(t) / 3
$$
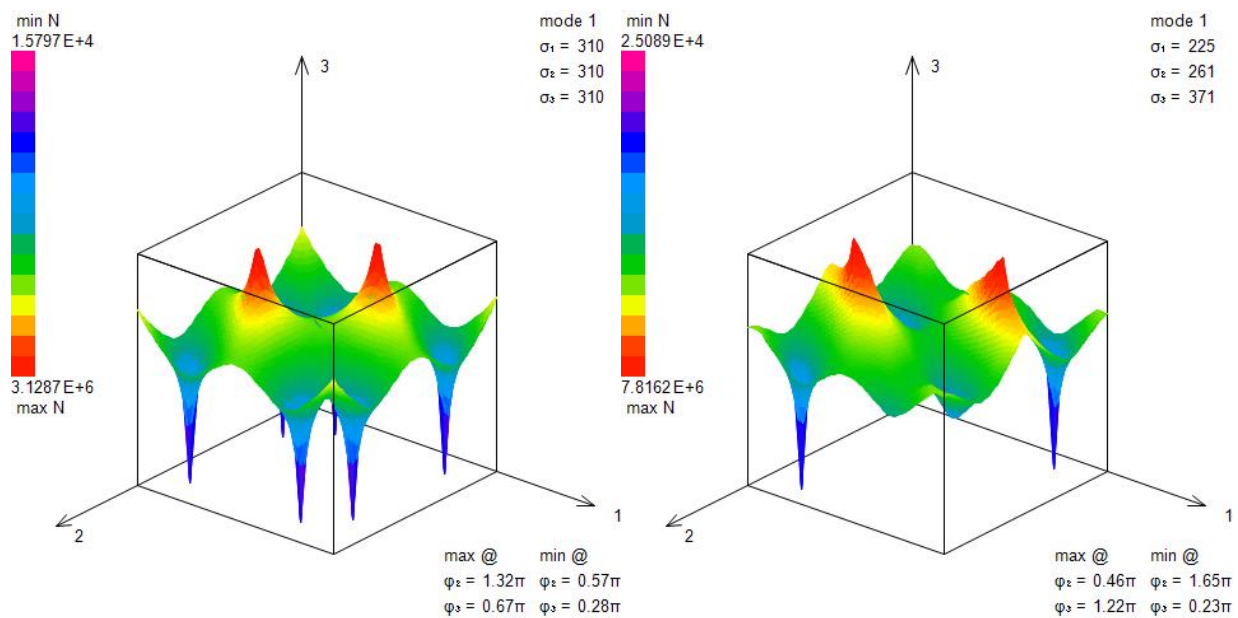

Fig.2.Fatigue life in terms of cycles $N$ as a function of shifts of phases $\varphi_{2}, \varphi_{3}$.

It is possible to evaluate the amount of cycles $N$ for arbitrary amplitude values of a multiaxial cyclic loading as a function of shifts of phases. The significant effect on fatigue life caused by a shift of phase was found by calculations and can be seen in fig. 2. a and b.

\subsection{Bi-axial bending-torsion test}

A similar analysis was conducted for the case of a widely used bending-torsion cyclic loading with an arbitrary shift of phases. Let us again assume a harmonic law for principal stresses variation in time with frequency $\omega$ and an arbitrary shift of phase $\varphi$ :

$$
\begin{gathered}
\tau_{23}(t)=\tau_{m}+\tau_{a} \cos \omega t \\
\sigma_{33}(t)=\sigma_{m}+\sigma_{a} \cos (\omega t+\varphi)
\end{gathered}
$$

where $\sigma_{m}, \tau_{m}$ are mean stress components and $\sigma_{a}, \tau_{a}$ are their amplitudes. Spreads of the stresses within a loading cycle are:

$$
\Delta \tau_{23}=\tau_{a}\left(\cos \omega t_{1}-\cos \omega t_{2}\right)=2 \tau_{a} \sin \left(\omega \frac{t_{2}-t_{1}}{2}\right) \sin \left(\omega \frac{t_{2}+t_{1}}{2}\right)
$$




$$
\Delta \sigma_{33}=\sigma_{a}\left(\cos \left(\omega t_{1}+\varphi\right)-\cos \left(\omega t_{2}+\varphi\right)\right)=2 \sigma_{a} \sin \left(\omega \frac{t_{2}-t_{1}}{2}\right) \sin \left(\omega \frac{t_{2}+t_{1}}{2}+\varphi\right)
$$

where time instants $t_{1}$ and $t_{2}$ correspond to minimum and maximum values of shear stress at the plane with unit normal vector $\mathbf{n} ; t_{1}, t_{2} \in[0, T], T=2 \pi / \omega$.

The value of range of shear stress may be written as:

$$
\begin{gathered}
\Delta \tau^{2}=4 \sin ^{2} \omega \frac{t_{2}-t_{1}}{2}\left[\tau_{a}^{2} X_{\tau} \sin ^{2} \omega \xi+\tau_{a} \sigma_{a} X_{\sigma \tau} \sin \omega \xi \sin (\omega \xi+\varphi)+\sigma_{a}^{2} X_{\sigma} \sin ^{2}(\omega \xi+\varphi)\right] \\
\frac{t_{1}+t_{2}}{2}=\xi, X_{\tau}=x_{2}+x_{3}-4 x_{2} x_{3}, X_{\sigma \tau}=2 \sqrt{x_{2} x_{3}}\left(1-2 x_{3}\right), X_{\sigma}=x_{3}\left(1-x_{3}\right)
\end{gathered}
$$

where

$$
\begin{gathered}
B_{S}=\tau_{a} \sigma_{a} X_{\sigma \tau} \sin \varphi+\sigma_{a}^{2} X_{\sigma} \sin 2 \varphi \\
B_{C}=\tau_{a}^{2} X_{\tau}+\tau_{a} \sigma_{a} X_{\sigma \tau} \cos \varphi+\sigma_{a}^{2} X_{\sigma} \cos 2 \varphi
\end{gathered}
$$

Equations to calculate the time stamps are:

$$
\begin{gathered}
t_{1}=\frac{T}{4}\left(-\frac{\operatorname{arctg}\left(B_{S} / B_{C}\right)}{\pi}+k-1\right), t_{2}=\frac{T}{4}\left(-\frac{\operatorname{arctg}\left(B_{S} / B_{C}\right)}{\pi}+k+1\right) \\
\omega \xi_{0}=-\frac{\operatorname{arctg}\left(B_{S} / B_{C}\right)}{2}+\frac{\pi k}{2}, k=1,2,3
\end{gathered}
$$

Corresponding values of the unit-vector's components $x_{i}=n_{i}^{2}$ at the time stamps $t_{1}$ and $t_{2}$ are:

$$
x_{3}=\frac{\tau_{a}^{2} \sin ^{2} \omega \xi_{0}+\sigma_{a}^{2} \sin ^{2}\left(\omega \xi_{0}+\varphi\right)}{2 \sigma_{a}^{2} \sin ^{2}\left(\omega \xi_{0}+\varphi\right)}, x_{2}=0, x_{1}=1-x_{2}-x_{3}
$$

In a similar way a time stamp within the loading cycle that determines the maximal value of $\alpha_{\infty} \sigma_{H, \max }$ was found:

$$
\sigma_{H, \max }=\sigma_{m}
$$



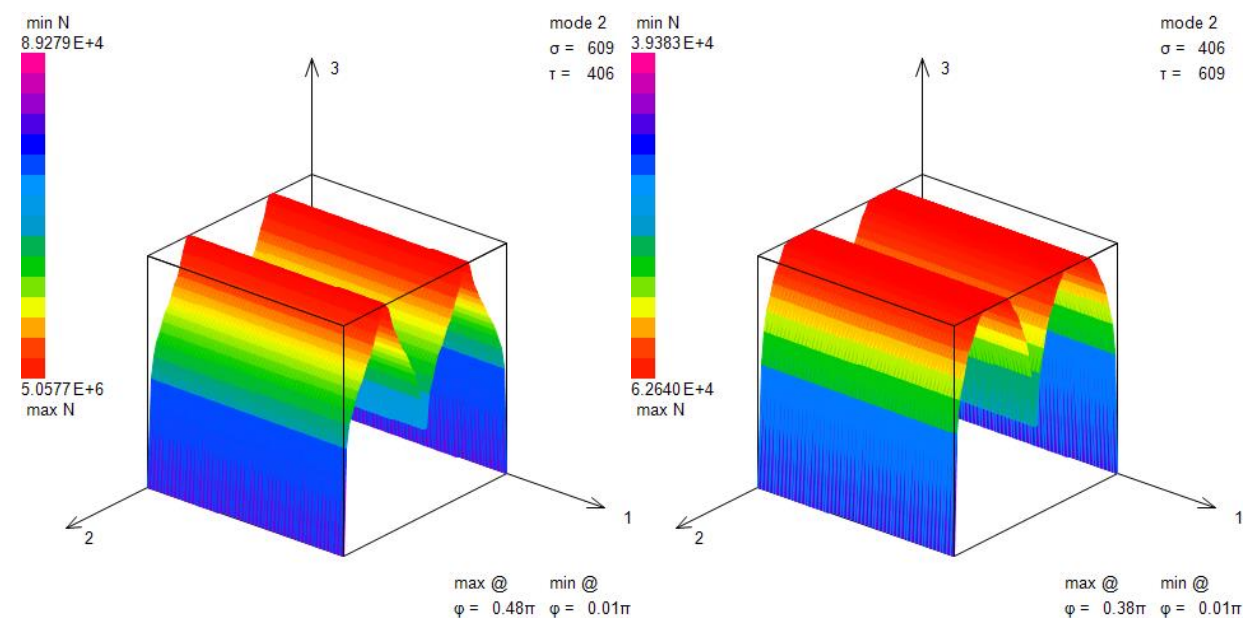

Fig. 3.Fatigue life in terms of cycles $N$ as a function of a shift of phase $\varphi$.

In the same way it is possible to evaluate amount of cycles $N$ to fracture for arbitrary amplitude values of a multiaxial cyclic loading as a function of a shift of phases. The significant effect on fatigue life that is caused by a shift of phase was found in calculations and can be seen in fig. 3 . a and $b$.

\section{Comparison of the criterion with experimental results}

For the disc with geometry presented in fig. 5-a and for stress components distribution presented in fig. 4 the amount of cycles $N$ before breakdown was estimated. The range of the value $N$ lays within $12 \cdot 10^{3}$ to $40 \cdot 10^{3}$ cycles. The results are similar to [9].
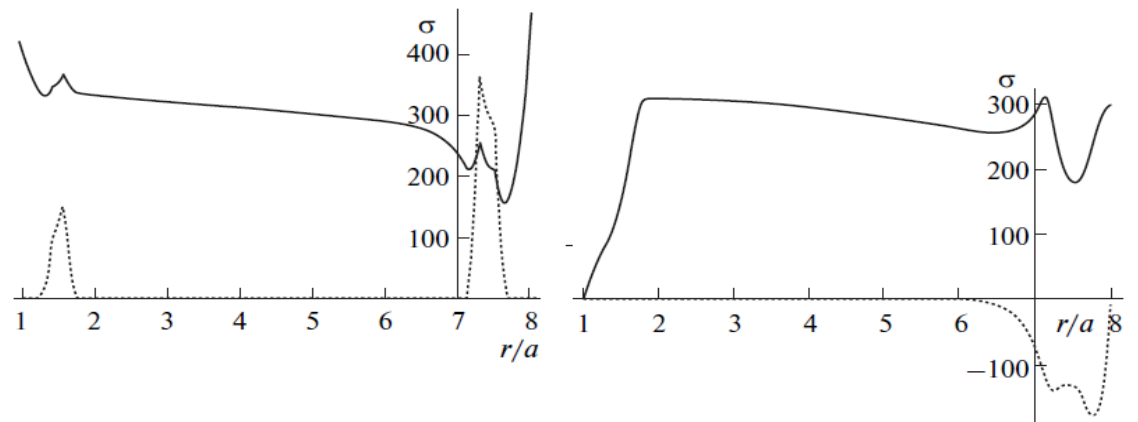

Fig. 4. Stress distribution within a simplified disc w/o v-shaped notches for blades.

In order to rate the criterion a comparison with other one [4] and experimental results [8] was made for bending-torsion experiments shown in Table 1: 
Table 1.Experimental and calculated data.

\begin{tabular}{rrrcccr}
\hline $\begin{array}{c}\sigma \\
(\mathrm{MPa})\end{array}$ & $\begin{array}{c}\tau \\
(\mathrm{MPa})\end{array}$ & $\delta$ & $\begin{array}{c}\alpha(\cdot \pi) \\
\text { experimental } \\
{[8]}\end{array}$ & $\begin{array}{c}\alpha(\cdot \pi) \\
\text { Carpinteri } \\
{[4]}\end{array}$ & $\begin{array}{c}\alpha(\cdot \pi) \\
\text { numerical } \\
{[2]}\end{array}$ & $\begin{array}{c}\alpha(\cdot \pi) \\
\text { analytical }\end{array}$ \\
\hline 0.00 & 201.11 & 0 & 0.249 & 0.250 & 0.250 & 0.250 \\
162.85 & 195.69 & 0 & 0.194 & 0.193 & 0.193 & 0.193 \\
274.68 & 137.34 & 0 & 0.128 & 0.125 & 0.125 & 0.125 \\
141.95 & 171.18 & $\pi / 6$ & 0.177 & 0.197 & 0.193 & 0.193 \\
255.06 & 127.53 & $\pi / 6$ & 0.090 & 0.119 & 0.125 & 0.125 \\
147.15 & 177.56 & $\pi / 3$ & 0.120 & 0.206 & 0.213 & 0.213 \\
255.06 & 127.53 & $\pi / 3$ & 0.045 & 0.094 & 0.125 & 0.125 \\
152.45 & 184.23 & $\pi / 2$ & 0.158 & 0.207 & 0.000 & 0.000 \\
264.87 & 132.44 & $\pi / 2$ & 0.000 & 0.076 & 0.000 & 0.000 \\
308.03 & 63.86 & $\pi / 2$ & 0.000 & 0.055 & 0.000 & 0.000 \\
\hline
\end{tabular}

\section{Numerical approach}

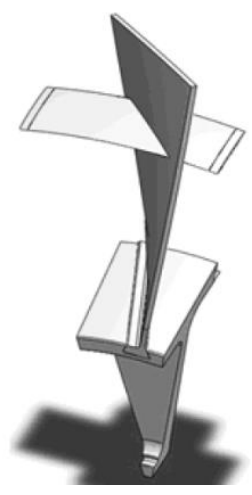

a

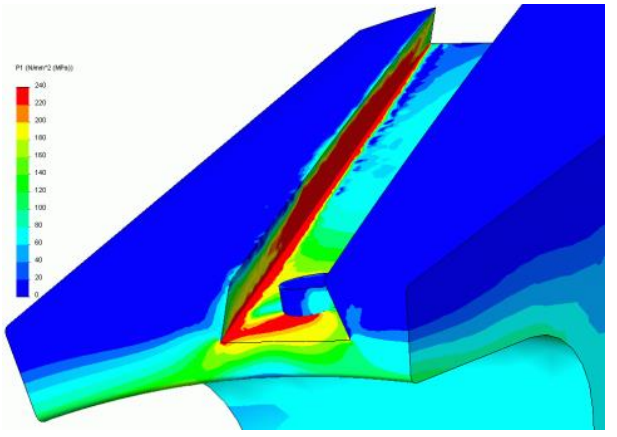

b

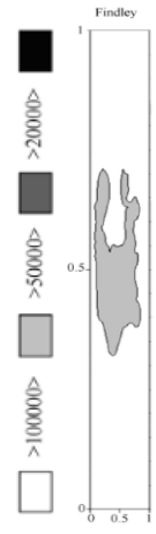

c

Fig. 5.Disc's fatigue life assessment; (a) - a segment of a compressor's disc and a blade, (b) - stress state within a contact zone between the disc and the blade, (c) - amount of cycles $N$ to breakdown within the most vulnerable zone of the disc.

Using the classic fatigue criterion [1] that takes into account a normal stress value impact on a critical plane orientation and can be written (see (27)) we conducted a finite element analysis for cases of in- and out-of-phase cyclic loadings.

$$
\left(\Delta \tau_{n} / 2+\alpha_{F} \sigma_{n}\right)_{M A X_{n}}=S_{F}+A_{F} N^{\beta_{F}}
$$

By calculating stress states of several engines' elements the values of fatigue durability $N$ were obtained. For instance in fig. 5-a a segment of compressor's disc and blade is shown. The blade undergoes aerodynamic and centrifugal forces. Fig. 5-b shows the stress state 
within a contact zone between the disc and the blade. Fig. 5-c shows the plot of amount of cycles $N$ to breakdown in the most loaded zone of the disc.

\section{Conclusions}

The analytical procedure to calculate the critical plane's orientation is proposed for the case of multiaxial cyclic loadings with an arbitrary shift of phases for the classic fatigue range low- and high-cycle fatigue. For the compressor's disc of a turbo shaft engine calculations such as determination of a stress state and locating a zone with the highest stress values were made. The assessment of amount of cycles $N$ to breakdown was given based on the calculations above.

\section{Acknowledgment}

The present work was supported by the Ministry of Science and Higher Education within the framework of the Russian State Assignment of ICAD RAS and IPMech RAS.

\section{References}

1. W. Findley. A theory for the effect of mean stress on fatigue of metals under combined torsion and axial load or bending. J. of Eng. for Indust.: 301-6 (1959)

2. I.V. Papadopoulos. Long life fatigue under multiaxial loading. International Journal of Fatigue, 23: 839-49 (2001)

3. F. Morel. A critical plane approach for life prediction of high cycle fatigue under multiaxial variable amplitude loading. Int. J. of Fatigue, 22(2): 101-19 (2000)

4. A. Carpinteri, A. Karolczuk, E. Macha, S. Vantadori. Expected position of the fatigue plane by using the weighted mean principal Euler angles. International Journal of Fatigue, 115: 87-99 (2002)

5. A. Banvillet, T. Palin-Luc, S. Lasserre. A volumetric energy based high cycle multiaxial fatigue criterion. International Journal of Fatigue, 25(8): 755-69 (2003)

6. N.G. Burago, A.B. Zhuravlev, I.S. Nikitin. Models of multiaxial fatigue fracture and service life estimation of structural elements. Mechanics of Solids, 46(6): 828-38 (2011)

7. I.S. Nikitin, N.G. Burago, A.D. Nikitin, V.L. Yakushev. Determination of the critical plane and assessment of fatigue durability under various cyclic loading regimes. PNRPU Mechanics Bulletin, 4: 238-52 (2017)

8. T. Nishihara, M. Kawamoto. The strength of metals under combined alternation bending and torsion with phase difference. Memories of the College of Engineering, Kyoto Imperial University, 11: 85-112 (1945)

9. N.G. Burago, I.S. Nikitin, P.A. Yushkovskiy. Lifetime of Disks of Variable Thickness with Anisotropy of Fatigue Properties Taken into Account. Mechanics of Solids, 50(5): 546-58 (2015) 